\title{
Evolutionary Variational Inequalities with Volterra Type Operators
}

\author{
Mykola Bokalo, Olha Sus* \\ Department of Mechanics and Mathematics, Ivan Franko National University of Lviv, Lviv, 79000, Ukraine
}

Received August 15, 2019; Revised September 19, 2019; Accepted September 27, 2019

Copyright (c) 2019 by authors, all rights reserved. Authors agree that this article remains permanently open access under the terms of the Creative Commons Attribution License 4.0 International License

\begin{abstract}
In this paper, we consider the initial-value problem for parabolic variational inequalities (subdifferential inclusions) with Volterra type operators. We prove the existence and the uniqueness of the solution. Furthermore, the estimates of the solution are obtained. The results are achieved using the Banach's fixed point theorem (the principle of compression mappings). The motivation for this work comes from the evolutionary variational inequalities arising in the study of frictionless contact problems for linear viscoelastic materials with long-term memory. Also, such kind of problems have their application in constructing different models of the injection molding processes.
\end{abstract}

Keywords Parabolic variational Inequality, Variational Inclusion, Volterra Type Operators

\section{Introduction}

We consider the problem for parabolic variational inequalities (subdifferential inclusions) with Volterra type operators. We start off by giving an example of the problem being studied here.

Let $n \in \mathbb{N}, p \geq 2, T>0, \Omega$ be a bounded domain in $\mathbb{R}^{n}, \partial \Omega$ be a boundary of $\Omega$. We put $Q:=\Omega \times(0, T), \Sigma:=\partial \Omega \times(0, T)$, $\Omega_{t}:=\Omega \times\{t\} \forall t \in[0, T], \Pi:=\{(t, s) \mid t \in(0, T), s \in$ $(0, t)\}$.

Let $L^{p}(\Omega)$ and $L^{p}(Q)$ be standard Lebesgue spaces. We denote by $W^{1, p}(\Omega)=\left\{v \in L^{p}(\Omega) \mid v_{x_{i}} \in L^{p}(\Omega), i=\right.$ $\overline{1, n}\}$ a standard Sobolev space with the norm $\|v\|_{W^{1, p}(\Omega)}:=$ $\left(\sum_{i=1}^{n}\left\|v_{x_{i}}\right\|_{L^{p}(\Omega)}^{p}++\|v\|_{L^{p}(\Omega)}^{p}\right)^{1 / p}$.

Let $K$ be a convex closed set in $W^{1, p}(\Omega)$ which contains 0 . Let us consider the problem of finding a function $u \in L^{p}(Q)$, $u_{x_{i}} \in L^{p}(Q), i=\overline{1, n}, u_{t} \in L^{2}(Q)$, that satisfies the initial condition

$$
\left.u\right|_{t=0}=u_{0}(x), \quad x \in \Omega,
$$

and, for a.e. $t \in(0, T], u(\cdot, t) \in K$ and

$$
\begin{gathered}
\int_{\Omega_{t}}\left\{u_{t}(v-u)+|\nabla u|^{p-2} \nabla u \nabla(v-u)+\right. \\
\left.+|u|^{p-2} u(v-u)+(v-u) \int_{0}^{t} \hat{b}(x, t, s) u(x, s) d s\right\} d x \geq \\
\geq \int_{\Omega_{t}} f(v-u) d x \quad \forall v \in K,
\end{gathered}
$$

where $f \in L^{2}(Q), u_{0} \in L^{2}(\Omega), \hat{b} \in L^{\infty}(\Omega \times(0, T) \times(0, T))$ are given, $\nabla u:=\left(u_{x_{1}}, \ldots, u_{x_{n}}\right)$.

Note that an operator $Q \ni(x, t) \mapsto \int_{0}^{t} \hat{b}(x, t, s) u(x, s) d s$ : $L^{2}(Q) \rightarrow L^{2}(Q)$ is called a Volterra type operator.

We remark that the problem (2) can be written in more abstract way. Indeed, after an appropriate identification of functions and functionals, one usually writes

$$
W^{1, p}(\Omega) \subset L^{2}(\Omega) \subset\left(W^{1, p}(\Omega)\right)^{\prime},
$$

where all the injections are continuous and dense (provided $W^{1, p}(\Omega)$ is reflexive). Clearly for any $h \in L^{2}(\Omega)$ and $v \in$ $W^{1, p}(\Omega)$ we have $\langle h, v\rangle=(h, v)$, where by $\langle\cdot, \cdot\rangle$ we denote the scalar product on dual pair $\left[\left(W^{1, p}(\Omega)\right)^{\prime}, W^{1, p}(\Omega)\right]$, and by $(\cdot, \cdot)$ we denote the scalar product in $L^{2}(\Omega)$. Thus, we may use the notation $(\cdot, \cdot)$ instead of $\langle\cdot, \cdot\rangle$.

Now we denote $V:=W^{1, p}(\Omega), H:=L^{2}(\Omega)$ and define operators $A: V \rightarrow V^{\prime}, \widehat{B}(t, s ; \cdot): H \rightarrow H,(t, s) \in \Pi$, as follows

$(A(v), w):=\int_{\Omega}\left[|\nabla v|^{p-2} \nabla v \nabla w+|v|^{p-2} v w\right] d x, \quad v, w \in V$, $(\widehat{B}(t, s) v)(\cdot):=\hat{b}(x, t, s) v(\cdot): \Omega \rightarrow \mathbb{R}, \quad(t, s) \in \Pi, v \in H$.

Then the problem is to find a function $u \in L^{p}(0, T ; V)$ such that $u^{\prime} \in L^{2}(0, T ; H), u(0)=u_{0}$ and, for a.e. $t \in(0, T)$, $u(t) \in K$ and

$$
\left(u^{\prime}(t)+A(u(t))+\int_{0}^{t} \widehat{B}(t, s) u(s) d s, v-u(t)\right) \geq
$$




$$
\geq(f(t), v-u(t)) \quad \forall v \in K,
$$

where $f \in L^{2}(0, T ; H), u_{0} \in H$ are given.

We remark that variational inequality (3) can be written as a subdifferential inclusion. For this purpose we put $I_{K}(v):=0$ if $v \in K$, and $I_{K}(v):=+\infty$ if $v \in V \backslash K$, and also

$$
\Phi(v):=\frac{1}{p} \int_{\Omega}\left[|\nabla v|^{p}+|v|^{p}\right] d x+I_{K}(v), \quad v \in V .
$$

It is easy to verify that the functional $\Phi: V \rightarrow \mathbb{R} \cup\{+\infty\}$ is convex and semi-lower-continuous. By the known results (see., e.g., [12, p. 83]) it follows that the problem of finding a solution of the variational inequality (3) can be written as such subdifferential inclusion: to find a function $u \in L^{p}(0, T ; V)$ such that $u^{\prime} \in L^{2}(0, T ; H), u(0)=u_{0}$ and, for a.e. $t \in(0, T)$, $u(t) \in D(\partial \Phi)$, and

$$
u^{\prime}(t)+\partial \Phi(u(t))+\int_{0}^{t} \widehat{B}(t, s) u(s) d s \ni f(t) \quad \text { in } \quad H
$$

where $\partial \Phi: V \rightarrow 2^{V^{\prime}}$ is a subdifferential of the functional $\Phi$.

The aim of the present paper is to investigate the problems for inclusions type (4).

The parabolic variational inequalities (subdifferential inclusions) and its modifications appear at the simulation of various physical processes, problems from economics, mechanics, finance, optimization, optimal control, game theory, and its research has been conducted in papers [12], [14], [19], [20], [21], [22], and others.

Let us remark that problems for evolutionary variational inequalities without Volterra type operators are widely investigated, particularly in [5], [8], [9], [15], [16], [23], [24], [25], [26], and others. Most of the results, which are known to us, were obtained by using the Banach's fixed point theorem (the principle of compression mappings).

Various classes of evolutionary variational inequalities with an elliptic principal parts and integral Volterra type operators have been investigated by many authors (in particular, see [27], [28], [29], [30]). For instance, in [30] it is obtained the mathematical model of the injection molding process which is characterized by the variational inequalities with an elliptic bilinear form and a Volterra term. Existence and uniqueness of the solution of these problems are derived by means of an argument based on the Banach's fixed point theorem.

In addition, in papers [28] and [29] it has been investigated an abstract evolutionary variational inequalities arising in the study of frictionless contact problems for linear viscoelastic materials with long-term memory. This abstract problem is of the following form:

find $u:[0, T] \rightarrow V$ which satisfies, for $t \in(0, T)$,

$$
\begin{gathered}
u(t) \in U, \\
(A u(t), v-u(t))_{V}+\left(\int_{0}^{t} B(t-s) u(s) d s, v-u(t)\right)_{V} \geq \\
\geq(f(t), v-u(t))_{V} \quad \forall v \in U, \quad t \in[0, T],
\end{gathered}
$$

where $V$ is a real Hilbert space, $T>0, U$ is a nonempty closed convex subset of $V, A: V \rightarrow V$ is a nonlinear operator, $B(t)$ : $V \rightarrow V$ is a linear continuous operator for all $t \in[0, T]$, and $f:[0, T] \rightarrow V$ is given. The results deal with the existence of a unique solution to problem, which is obtained by using arguments for time-dependent elliptic variational inequalities.

In this paper, we consider the problem for parabolic variational inequalities with Volterra type operators, that is, when the value of the solution in the actual moment of time depends on the values of the solution in the previous moments.

Initial-boundary value problems for nonlinear evolutionary equations with Volterra type operators, which can be interpreted as a partial case of evolutionary variational inequalities, are investigated in papers [17], [18]. Let us note that problems for parabolic variational inequalities or subdifferential inclusions with Volterra type operators have not been considered in the literature before. It serves us as one of the motivations for the study of such kind of problems.

The paper is structured as follows. In Section 1, we formulate the main notations and auxiliary facts. The main results of our paper are established in Section 2. In Section 3, it is substantiated the main results.

\section{Preliminaries}

Let $V$ be a separable reflexive Banach space with the norm $\|\cdot\|, H$ be the Hilbert space with the scalar product $(\cdot, \cdot)$ and the norm $|\cdot|$. Assume that the injection $V \subset H$ is dense, continuous, and compact.

Let $V^{\prime}$ and $H^{\prime}$ be the dual spaces to $V$ and $H$, respectively. We assume (after an appropriate identification of functionals), that the space $H^{\prime}$ is a subspace of $V^{\prime}$. Identifying (by the Riesz-Fréchet representation theorem) $H^{\prime}$ with $H$, one usually writes

$$
V \subset H \subset V^{\prime}
$$

where all the injections are continuous and dense (provided $\mathrm{V}$ is reflexive).

Note that in this case $\langle g, v\rangle_{V}=(g, v)$ for every $v \in V$, $g \in H$, where $\langle\cdot, \cdot\rangle_{V}$ is the scalar product on the duality pair $\left[V^{\prime}, V\right]$. Therefore, further we will use the notation $(\cdot, \cdot)$ instead of $\langle\cdot, \cdot\rangle_{V}$.

Let $T>0$ be an arbitrary fixed number. Let us introduce some spaces of functions and spaces of distributions. Let $X$ be an arbitrary Banach space with the norm $\|\cdot\|_{X}$. By $C([0, T] ; X)$ we mean the Banach space of continuous functions $w:[0, T] \rightarrow X$ with the norm $\|w\|_{C(0, T ; X)}:=$ $\max _{t \in[0, T]}\|w(t)\|_{X}$. By $L^{q}(0, T ; X)$, where $q \geq 1$, we denote the Banach space of measurable functions $w:(0, T) \rightarrow X$ such that $\|w(\cdot)\|_{X} \in L^{q}(0, T)$ with the norm $\|w\|_{L^{q}(0, T ; X)}:=$ $\left(\int_{0}^{T}\|w(t)\|^{q} d t\right)^{1 / q}$. By $L^{\infty}(0, T ; X)$ we denote the Banach space of measurable and essentially bounded functions $w:(0, T) \rightarrow X$ such that $\|w(\cdot)\|_{X} \in L^{\infty}(0, T)$ with the norm $\|w\|_{L^{\infty}(0, T ; X)}:=\operatorname{ess} \sup _{t \in(0, T)}\|w(t)\|_{X}$. By $D^{\prime}(0, T ; X)$ we mean the space of distributions on $D(0, T)$ with values on $X$, i.e., the space of linear continuous (in weak topology on $X$ ) 
functionals on $D(0, T)$ with values on $X$ (hereafter $D(0, T)$ is space of test functions, that is, the space of infinitely differentiable on $(0, T)$ functions with compact supports, equipped with the corresponding weak topology). It is easy to see (using (5)), that spaces $L^{q}(0, T ; V), L^{2}(0, T ; H), L^{q^{\prime}}\left(0, T ; V^{\prime}\right)$, where $q>1,1 / q+1 / q^{\prime}=1$, can be identified with the corresponding subspaces of $D^{\prime}\left(0, T ; V^{\prime}\right)$. In particular, this allows us to talk about derivatives $w^{\prime}$ of functions $w$ from $L^{q}(0, T ; V)$ and $L^{2}(0, T ; H)$ in the sense of distributions $D^{\prime}\left(0, T ; V^{\prime}\right)$ and belonging of such derivatives to $L^{q^{\prime}}\left(0, T ; V^{\prime}\right)$ or $L^{2}(0, T ; H)$.

Let us define the spaces

$$
\begin{aligned}
H^{1}(0, T ; H) & :=\left\{w \in L^{2}(0, T ; H) \mid w^{\prime} \in L^{2}(0, T ; H)\right\}, \\
W_{q}^{1}(0, T ; V) & :=\left\{w \in L^{q}(0, T ; V) \mid w^{\prime} \in L^{q^{\prime}}\left(0, T ; V^{\prime}\right)\right\},
\end{aligned}
$$

$q>1, q^{\prime}:=q /(q-1)$.

From known results (see., for example, [10, p. 177-179]) it follows that $H^{1}(0, T ; H) \subset C([0, T] ; H)$ and $W_{q}^{1}(0, T ; V) \subset$ $C([0, T] ; H)$. Moreover, for every $w$ in $H^{1}(0, T ; H)$ or $W_{q}^{1}(0, T ; V)$ the function $t \mapsto|w(t)|^{2}$ is absolutely continuous on $[0, T]$ and the following equality holds

$$
\frac{d}{d t}|w(t)|^{2}=2\left(w^{\prime}(t), w(t)\right) \quad \text { for a.e. } \quad t \in[0, T] .
$$

\section{Statement of the problem and the main results}

Let $\Phi: V \rightarrow(-\infty,+\infty]$ be a proper functional, i.e., $\operatorname{dom}(\Phi):=\{v \in V \mid \Phi(v)<+\infty\} \neq \varnothing$, which satisfies the following conditions:

$$
\begin{aligned}
& \left(\mathcal{A}_{1}\right) \quad \Phi(\alpha v+(1-\alpha) w) \leq \alpha \Phi(v)+(1-\alpha) \Phi(w) \\
& \forall v, w \in V, \forall \alpha \in[0,1], \\
& \text { i.e., the functional } \Phi \text { is convex, }
\end{aligned}
$$$$
\left(\mathcal{A}_{2}\right) \quad v_{k} \underset{k \rightarrow \infty}{\longrightarrow} v \text { in } V \Longrightarrow \varliminf_{k \rightarrow \infty} \Phi\left(v_{k}\right) \geq \Phi(v) \text {, }
$$

i.e., the functional $\Phi$ is lower semicontinuous.

Recall that the subdifferential of functional $\Phi$ is a mapping $\partial \Phi: V \rightarrow 2^{V^{\prime}}$, defined as follows

$$
\partial \Phi(v):=\left\{v^{*} \in V^{\prime} \mid \Phi(w) \geq \Phi(v)+\left(v^{*}, w-v\right) \quad \forall w \in V\right\},
$$

$v \in V$, and the domain of the subdifferential $\partial \Phi$ is the set $D(\partial \Phi):=\{v \in V \mid \partial \Phi(v) \neq \varnothing\}$. We identify the subdifferential $\partial \Phi$ with its graph, assuming that $\left[v, v^{*}\right] \in \partial \Phi$ if and only if $v^{*} \in \partial \Phi(v)$, i.e., $\partial \Phi=\left\{\left[v, v^{*}\right] \mid v \in D(\partial \Phi), v^{*} \in\right.$ $\partial \Phi(v))\}$. R. Rockafellar (see [11, theorem A]) proves that the subdifferential $\partial \Phi$ is a maximal monotone operator, that is,

$$
\left(v_{1}^{*}-v_{2}^{*}, v_{1}-v_{2}\right) \geq 0 \quad \forall\left[v_{1}, v_{1}^{*}\right],\left[v_{2}, v_{2}^{*}\right] \in \partial \Phi,
$$

and for every element $\left[v_{1}, v_{1}^{*}\right] \in V \times V^{\prime}$ we have the implication

$$
\left(v_{1}^{*}-v_{2}^{*}, v_{1}-v_{2}\right) \geq 0 \quad \forall\left[v_{2}, v_{2}^{*}\right] \in \partial \Phi .
$$

From this implies that $\left[v_{1}, v_{1}^{*}\right] \in \partial \Phi$.

Additionally, assume that the following condition hold:
$\left(\mathcal{A}_{3}\right)$ there exist constants $p \geq 2, K>0$ such that

$$
\Phi(v) \geq K\|v\|^{p} \quad \forall v \in \operatorname{dom}(\Phi) ;
$$

moreover, $\Phi(0)=0$.

Remark 2. Condition $\left(\mathcal{A}_{3}\right)$ implies that $\Phi(v) \geq \Phi(0)+$ $(0, v-0) \forall v \in V$, hence $[0,0] \in \partial \Phi$.

Let $\mathcal{B}: L^{2}(0, T ; H) \rightarrow L^{2}(0, T ; H)$ be an operator that satisfies the following condition:

$\left(\mathcal{A}_{4}\right)$ there exists a constant $L \geq 0$ such that, for a.e. $t \in$ $(0, T)$ and for any $w_{1}, w_{2} \in L^{2}(0, T ; H)$, the following inequality holds:

$$
\left|\mathcal{B}\left(w_{1}\right)(t)-\mathcal{B}\left(w_{2}\right)(t)\right| \leq L \int_{0}^{t}\left|w_{1}(s)-w_{2}(s)\right| d s
$$

moreover, $\mathcal{B}(0)=0$.

It is easy to varify that the condition $\left(\mathcal{A}_{4}\right)$ implies that

$$
|\mathcal{B}(w)(t)| \leq L \int_{0}^{t}|w(s)| d s
$$

for a.e. $t \in(0, T)$ and for any $w \in L^{2}(0, T ; H)$.

Operator $\mathcal{B}$ is called a Volterra type operator.

Remark 3. An important example of $\mathcal{B}$ is the following operator:

$$
\mathcal{B}(w)(t):=B\left(t, \int_{0}^{t} b(t, s, w(s)) d s\right), \quad t \in(0, T),
$$

where $B:(0, T) \times H \rightarrow H, b: \Pi \times H \rightarrow H$ are mappings which satisfy conditions:

- for any $v \in H$ the mapping $B(\cdot, v):(0, T) \rightarrow H$ is measurable, and there exists a constant $L_{1} \geq 0$ such that the following inequality holds

$$
\left|B\left(t, v_{1}\right)-B\left(t, v_{2}\right)\right| \leq L_{1}\left|v_{1}-v_{2}\right|
$$

for a.e. $t \in(0, T)$ and for all $v_{1}, v_{2} \in H$; in addition, $B(t, 0)=0$ for a.e. $t \in(0, T)$.

- for any $v \in H$ the mapping $b(\cdot, \cdot, v): \Pi \rightarrow H$ is measurable, and there exists a constant $L_{2} \geq 0$ such that the following inequality holds

$$
\left|b\left(t, s, v_{1}\right)-b\left(t, s, v_{2}\right)\right| \leq L_{2}\left|v_{1}-v_{2}\right|
$$

for a.e. $(t, s) \in \Pi$ and for all $v_{1}, v_{2} \in H$; in addition, $b(t, s, 0)=0$ for a.e. $(t, s) \in \Pi$.

Let us show that the operator $\mathcal{B}$, which is defined in (9), satisfies the condition $\left(\mathcal{A}_{4}\right)$ with $L=L_{1} L_{2}$. Indeed, based on (10) and (11) we have

$$
\left|\mathcal{B}\left(w_{1}\right)(t)-\mathcal{B}\left(w_{2}\right)(t)\right| \leq
$$




$$
\begin{gathered}
\leq\left|B\left(t, \int_{0}^{t} b\left(t, s, w_{1}(s)\right) d s\right)-B\left(t, \int_{0}^{t} b\left(t, s, w_{2}(s)\right) d s\right)\right| \leq \\
\leq L_{1}\left|\int_{0}^{t} b\left(t, s, w_{1}(s)\right) d s-\int_{0}^{t} b\left(t, s, w_{2}(s)\right) d s\right| \leq \\
\leq L_{1} L_{2} \int_{0}^{t}\left|w_{1}(s)-w_{2}(s)\right| d s .
\end{gathered}
$$

Let us consider the evolutionary variational inequality (subdifferential inclusion)

$$
u^{\prime}(t)+\partial \Phi(u(t))+\mathcal{B}(u)(t) \ni f(t), \quad t \in(0, T),
$$

where $f:(0, T) \rightarrow V^{\prime}$ is a given measurable function, $u$ : $(0, T) \rightarrow V$ is an unknown function.

Definition. Let conditions $\left(\mathcal{A}_{1}\right)-\left(\mathcal{A}_{4}\right)$ hold, and $f \in$ $L^{p^{\prime}}\left(0, T ; V^{\prime}\right)$, where $p^{\prime}=p /(p-1)$. The solution of variational inequality (12) is called a function $u$ that satisfies the following conditions

1) $u \in W_{p}^{1}(0, T ; V)$ (then $\left.u \in C([0, T] ; H)\right)$;

2) $u(t) \in D(\partial \Phi)$ for a.e. $t \in(0, T)$;

3) there exists a function $g \in L^{p^{\prime}}\left(0, T ; V^{\prime}\right)$ such that, for a.e. $t \in(0, T)$, we have $g(t) \in \partial \Phi(u(t))$ and

$$
u^{\prime}(t)+g(t)+\mathcal{B}(u)(t)=f(t) \text { in } V^{\prime} .
$$

We consider the problem of finding a solution $u$ of the variational inequality (12) that satisfies the following condition

$$
u(0)=u_{0},
$$

where $u_{0} \in H$ is given.

It is called the problem $\mathbf{P}\left(\Phi, \mathcal{B}, f, u_{0}\right)$, and the function $u$ is called its solution.

Remark 4. The problem $\mathbf{P}\left(\Phi, \mathcal{B}, f, u_{0}\right)$ can be replaced by the following one. Let $K$ be a convex, closed set in $V$, $A: V \rightarrow V^{\prime}$ be a monotone, bounded and semi-continuous operator such that $(A(v), v) \geq \widetilde{K}_{1}\|v\|^{p} \quad \forall v \in V$, where $\widetilde{K}_{1}=$ const $>0$, and $f \in L^{p^{\prime}}\left(0, T ; V^{\prime}\right), u_{0} \in H$. The problem is to find a function $u \in W_{p}^{1}(0, T ; V)$ such that $u(0)=u_{0}$ and, for a.e. $t \in(0, T)$, we have $u(t) \in K$ and $\left(u^{\prime}(t)+A(u(t))+\mathcal{B}(u)(t), v-u(t)\right) \geq(f(t), v-u(t))$ $\forall v \in K$.

Theorem 1 (uniqueness of the solution). Let conditions $\left(\mathcal{A}_{1}\right)-\left(\mathcal{A}_{4}\right)$ hold, and $f \in L^{p^{\prime}}\left(0, T ; V^{\prime}\right), u_{0} \in H$. Then the problem $\boldsymbol{P}\left(\Phi, \mathcal{B}, f, u_{0}\right)$ has no more than one solution.

Theorem 2 (existence of the solution). Let conditions $\left(\mathcal{A}_{1}\right)$ - $\left(\mathcal{A}_{4}\right)$ hold, and $f \in L^{2}(0, T ; H), u_{0} \in \operatorname{dom}(\Phi)$. Then the problem $\boldsymbol{P}\left(\Phi, \mathcal{B}, f, u_{0}\right)$ has a unique solution, it belongs to the space $L^{\infty}(0, T ; V) \cap H^{1}(0, T ; H)$, while $\Phi(u(\cdot)) \in$ $C([0, T] ; H)$, and satisfies estimates

$$
\max _{t \in[0, T]}|u(t)|^{2}+\int_{0}^{T}\|u(t)\|^{p} d t+\int_{0}^{T} \Phi(u(t)) d t \leq
$$

$$
\begin{gathered}
\leq C_{1}\left(\left|u_{0}\right|^{2}+\int_{0}^{T}|f(t)|^{2} d t\right) \\
\operatorname{essip}_{t \in[0, T]}\|u(t)\|^{p}+\max _{t \in[0, T]} \Phi(u(t))+\int_{0}^{T}\left|u^{\prime}(t)\right|^{2} d t \leq \\
\leq C_{2}\left(\left|u_{0}\right|^{2}+\Phi\left(u_{0}\right)+\int_{0}^{T}|f(t)|^{2} d t\right),
\end{gathered}
$$

where $C_{1}, C_{2}$ are positive constants depending on $K, L$, and $T$ only.

\section{Proof of the main results}

Proof. Proof of Theorem 1. Assume the contrary. Let $u_{1}$ and $u_{2}$ are two solutions of the problem $\mathbf{P}\left(\Phi, \mathcal{B}, f, u_{0}\right)$. Then for every $i \in\{1,2\}$ there exists function $g_{i} \in L^{p^{\prime}}\left(0, T ; V^{\prime}\right)$ such that, for a.e. $t \in(0, T)$, we have $g_{i}(t) \in \partial \Phi\left(u_{i}(t)\right)$ and

$$
u_{i}^{\prime}(t)+g_{i}(t)+\mathcal{B}\left(u_{i}\right)(t)=f(t) \quad \text { in } V^{\prime}, \quad i=1,2 .
$$

We put $w:=u_{1}-u_{2}$. From equalities (17), for a.e. $t \in(0, T)$, we obtain

$$
w^{\prime}(t)+g_{1}(t)-g_{2}(t)+\mathcal{B}\left(u_{1}\right)(t)-\mathcal{B}\left(u_{2}\right)(t)=0 \text { in } V^{\prime} .
$$

Multiplying equality (18) for a.e. $t \in(0, T)$ by $w(t)$, we obtain

$$
\begin{gathered}
\left(w^{\prime}(t), w(t)\right)+\left(g_{1}(t)-g_{2}(t), u_{1}(t)-u_{2}(t)\right)+ \\
+\left(\mathcal{B}\left(u_{1}\right)(t)-\mathcal{B}\left(u_{2}\right)(t), w(t)\right)=0 .
\end{gathered}
$$

By (6), (7) and the fact that $\left[u_{i}(t), g_{i}(t)\right] \in \partial \Phi$, for a.e. $t \in$ $(0, T), i=1,2$, we obtain

$$
\frac{1}{2} \frac{d|w(t)|^{2}}{d t}+\left(\mathcal{B}\left(u_{1}\right)(t)-\mathcal{B}\left(u_{2}\right)(t), w(t)\right) \leq 0
$$

for a.e. $t \in(0, T)$.

Integrating (20) from 0 to $\tau$, where $\tau$ is an arbitrary from $(0, T]$, and taking into account that $w(0)=0$, we obtain

$$
|w(\tau)|^{2}+2 \int_{0}^{\tau}\left(\mathcal{B}\left(u_{1}\right)(t)-\mathcal{B}\left(u_{2}\right)(t), w(t)\right) d t \leq 0 .
$$

Let move the second term of inequality (21) to the right hand side. By the condtion $\left(\mathcal{A}_{4}\right)$ and the Cauchy-Schwartz inequality we obtain

$$
\begin{aligned}
& \left|\int_{0}^{\tau}\left(\mathcal{B}\left(u_{1}\right)(t)-\mathcal{B}\left(u_{2}\right)(t), w(t)\right) d t\right| \leq \\
& \leq \int_{0}^{\tau}\left|\mathcal{B}\left(u_{1}\right)(t)-\mathcal{B}\left(u_{2}\right)(t)\right||w(t)| d t \leq \\
& \quad \leq L \int_{0}^{\tau}\left(\int_{0}^{t}|w(s)| d s\right)|w(t)| d t \leq
\end{aligned}
$$




$$
\begin{gathered}
\leq L \sqrt{T}\left(\int_{0}^{\tau}|w(t)|^{2} d t\right)^{1 / 2}\left(\int_{0}^{\tau}\left(\int_{0}^{t}|w(s)|^{2} d s\right) d t\right)^{1 / 2} \leq \\
\leq L T \int_{0}^{\tau}|w(t)|^{2} d t .
\end{gathered}
$$

Thus, based on (22) from (21) we obtain

$$
|w(\tau)|^{2} \leq 2 L T \int_{0}^{\tau}|w(t)|^{2} d t .
$$

Using the Gronwall-Belman lemma, from (23) we get $|w(\tau)|^{2} \leq 0, \tau \in[0, T]$. From this it follows $w(\tau)=$ $0 \forall \tau \in[0, T]$, i.e., $u_{1}=u_{2}$. This contradicts our assumption. Therefore, a solution of the problem $\mathbf{P}\left(\Phi, \mathcal{B}, f, u_{0}\right)$ is unique.

Proof. Proof of Theorem 2. First, we define the functional $\Phi_{H}: H \rightarrow(-\infty ;+\infty]$ by the rule: $\Phi_{H}(v):=\Phi(v)$, if $v \in V$, and $\Phi_{H}(v):=+\infty$ otherwise. Note that conditions $\left(\mathcal{A}_{1}\right),\left(\mathcal{A}_{2}\right)$, Lemma IV.5.2 and Proposition IV.5.2 of the monograph [12] imply that $\Phi_{H}$ is a proper, convex and lower-semicontinuous functional on $H, \operatorname{dom}\left(\Phi_{H}\right)=\operatorname{dom}(\Phi) \subset V$, and $\partial \Phi_{H}=\partial \Phi \cap(V \times H)$, where $\partial \Phi_{H}: H \rightarrow 2^{H}$ is the subdifferential of the functional $\Phi_{H}$. Moreover, condition $\left(\mathcal{A}_{3}\right)$ yields $0 \in \partial \Phi_{H}(0)$.

Let us consider the auxiliary problem of finding a function $u \in H^{1}(0, T ; H)$ such that $u(0)=u_{0}$ and, for a.e. $t \in(0, T)$, inclusions $u(t) \in D\left(\partial \Phi_{H}\right)$, and

$$
u^{\prime}(t)+\partial \Phi_{H}(u(t))+\mathcal{B}(u)(t) \ni f(t) \quad \text { in } H
$$

hold, i.e., there exists a function $g \in L^{2}(0, T ; H)$ such that, for a.e. $t \in(0, T)$, we have $g(t) \in \partial \Phi_{H}(u(t))$, and

$$
u^{\prime}(t)+g(t)+\mathcal{B}(u)(t)=f(t) \quad \text { in } H .
$$

This problem is called $\mathbf{P}\left(\Phi_{H}, \mathcal{B}, f, u_{0}\right)$.

Let us show that there exists a unique solution of the problem $\mathbf{P}\left(\Phi_{H}, \mathcal{B}, f, u_{0}\right)$. For this we needed the following statements.

Proposition 1 ([12, Lemma IV.4.3]). Let $v \in H^{1}(0, T ; H)$ and $\widehat{g} \in L^{2}(0, T ; H)$ such that, for a.e. $t \in(0, T), v(t) \in$ $D\left(\partial \Phi_{H}\right)$ and $\widehat{g}(t) \in \partial \Phi_{H}(v(t))$. Then the function $\Phi_{H}(v(\cdot))$ is absolutely continuous on the interval $[0, T]$ and for any function $h:[0, T] \rightarrow H$ such that $h(t) \in \partial \Phi_{H}(v(t))$ the following equality holds

$$
\frac{d}{d t} \Phi_{H}(v(t))=\left(h(t), v^{\prime}(t)\right) \quad \text { for a.e. } t \in(0, T) .
$$

Proposition 2 ([7, Proposition 3.12], [12, Proposition IV.5.2]). Let $h \in L^{2}(0, T ; H)$ and $w_{0} \in \operatorname{dom}(\Phi)$. Then there exists a unique function $w \in H^{1}(0, T ; H)$ such that $w(0)=w_{0}$ and, for a.e. $t \in(0, T), w(t) \in D\left(\partial \Phi_{H}\right)$ and

$$
w^{\prime}(t)+\partial \Phi_{H}(w(t)) \ni h(t) \text { in } H,
$$

i.e., there exists a function $\widetilde{g} \in L^{2}(0, T ; H)$ such that, for a.e. $t \in(0, T), \widetilde{g}(t) \in \partial \Phi_{H}(w(t))$, and

$$
w^{\prime}(t)+\widetilde{g}(t)=h(t) \text { in } H
$$

Let us continue to prove our theorem.

Let $M:=\left\{w \in C([0, T] ; H) \mid w(0)=u_{0}\right\}$ be a set of functions with a metric

$$
\rho\left(u_{1}, u_{2}\right)=\max _{t \in[0, T]}\left[e^{-\alpha t}\left|u_{1}(t)-u_{2}(t)\right|\right], \quad u_{1}, u_{2} \in M,
$$

where $\alpha>0$ is an arbitrary fixed number. It is obvious that the metric space is complete.

Now let us consider an operator $A: M \rightarrow M$ which is defined as follows: for any given function $\widetilde{w} \in M$, it defines a function $w \in M$ such that $w \in H^{1}(0, T ; H), w(0)=u_{0}$ and, for a.e. $t \in(0, T), w(t) \in D\left(\Phi_{H}\right)$ and

$$
w^{\prime}(t)+\partial \Phi_{H}(w(t)) \ni f(t)-\mathcal{B}(\widetilde{w})(t) \text { in } H .
$$

Clearly, variational inequality (28) coincides with variational inequality (26) after replacing $h$ by $f-\mathcal{B}(\widetilde{w})$. Thus, using Proposition 2 we get that operator $A$ is well-defined. Let us show that the operator $A$ is a contraction operator. Indeed, let $\tilde{w}_{1}, \tilde{w}_{2}$ be arbitrary from $M$ and $w_{1}:=A \widetilde{w}_{1}, w_{2}:=A \widetilde{w}_{2}$. According to (28) there exist $g_{1}, g_{2} \in L^{2}(0, T ; H)$ such that for each $k \in\{1,2\}$ and for a.e. $t \in(0, T)$ we have $g_{k}(t) \in$ $\partial \Phi_{H}\left(w_{k}(t)\right)$ and

$$
w_{k}^{\prime}(t)+g_{k}(t)=f(t)-\mathcal{B}\left(\widetilde{w}_{k}\right)(t) \text { in } H .
$$

Subtracting identity (29) for $k=2$ from identity (29) for $k=1$, and, for a.e. $t \in(0, T)$, multiplying the obtained identity by $w_{1}(t)-w_{2}(t)$, we get

$$
\begin{gathered}
\left(\left(w_{1}(t)-w_{2}(t)\right)^{\prime}, w_{1}(t)-w_{2}(t)\right)+ \\
+\left(g_{1}(t)-g_{2}(t), w_{1}(t)-w_{2}(t)\right)= \\
=-\left(\mathcal{B}\left(\widetilde{w}_{1}\right)(t)-\mathcal{B}\left(\widetilde{w}_{2}\right)(t), w_{1}(t)-w_{2}(t)\right), \\
w_{1}(0)-w_{2}(0)=0 .
\end{gathered}
$$

We integrate equality (30) by $t$ from 0 to $\tau \in(0, T]$, taking into account that for a.e. $t \in(0, T)$ we have

$$
\left(\left(w_{1}(t)-w_{2}(t)\right)^{\prime}, w_{1}(t)-w_{2}(t)\right)=\frac{1}{2} \frac{d}{d t}\left|w_{1}(t)-w_{2}(t)\right|^{2} .
$$

As a result we obtain the equality

$$
\begin{gathered}
\frac{1}{2}\left|w_{1}(\tau)-w_{2}(\tau)\right|^{2}+\int_{0}^{\tau}\left(g_{1}(t)-g_{2}(t), w_{1}(t)-w_{2}(t)\right) d t= \\
=-\int_{0}^{\tau}\left(\mathcal{B}\left(\widetilde{w}_{1}\right)(t)-\mathcal{B}\left(\widetilde{w}_{2}\right)(t), w_{1}(t)-w_{2}(t)\right) d t .
\end{gathered}
$$

By condition $\left(\mathcal{A}_{2}\right)$, for a.e. $t \in(0, T)$, we obtain

$$
\left(g_{1}(t)-g_{2}(t), w_{1}(t)-w_{2}(t)\right) \geq 0 .
$$


We will use the Cauchy inequality

$$
a b \leq \varepsilon a^{2}+(4 \varepsilon)^{-1} b^{2} \quad \forall a, b \in \mathbb{R}, \quad \forall \varepsilon>0 .
$$

According to $\left(\mathcal{A}_{4}\right)$ and (34) with $\varepsilon=1$, for a.e. $t \in(0, T)$, we obtain

$$
\begin{gathered}
\left|\left(\mathcal{B}\left(\widetilde{w}_{1}\right)(t)-\mathcal{B}\left(\widetilde{w}_{2}\right)(t), w_{1}(t)-w_{2}(t)\right)\right| \leq \\
\leq\left|\mathcal{B}\left(\widetilde{w}_{1}\right)(t)-\mathcal{B}\left(\widetilde{w}_{2}\right)(t)\right|\left|w_{1}(t)-w_{2}(t)\right| \leq \\
\leq L\left(\int_{0}^{t}\left|\widetilde{w}_{1}(s)-\widetilde{w}_{2}(s)\right| d s\right)\left|w_{1}(t)-w_{2}(t)\right| \leq \\
\leq\left|w_{1}(t)-w_{2}(t)\right|^{2}+4^{-1} L^{2}\left(\int_{0}^{t}\left|\widetilde{w}_{1}(s)-\widetilde{w}_{2}(s)\right| d s\right)^{2} \leq \\
\leq\left|w_{1}(t)-w_{2}(t)\right|^{2}+4^{-1} L^{2} T \int_{0}^{t}\left|\widetilde{w}_{1}(s)-\widetilde{w}_{2}(s)\right|^{2} d s . \quad(35)
\end{gathered}
$$

From (32), according to (33) and (35), we have

$$
\begin{gathered}
\left|w_{1}(\tau)-w_{2}(\tau)\right|^{2} \leq 2 \int_{0}^{\tau}\left|w_{1}(t)-w_{2}(t)\right|^{2} d t+ \\
+2^{-1} L^{2} T \int_{0}^{\tau}\left(\int_{0}^{t}\left|\widetilde{w}_{1}(s)-\widetilde{w}_{2}(s)\right|^{2} d s\right) d t .
\end{gathered}
$$

Let us evaluate the right-hand side of inequality(36) in the following way

$$
\int_{0}^{\tau}\left(\int_{0}^{t}\left|\widetilde{w}_{1}(s)-\widetilde{w}_{2}(s)\right|^{2} d s\right) d t \leq T \int_{0}^{\tau}\left|\widetilde{w}_{1}(s)-\widetilde{w}_{2}(s)\right|^{2} d s .
$$

From (36), taking into account (37), we get

$$
\begin{gathered}
\left|w_{1}(\tau)-w_{2}(\tau)\right|^{2} \leq 2 \int_{0}^{\tau}\left|w_{1}(t)-w_{2}(t)\right|^{2} d t+ \\
+2^{-1}(L T)^{2} \int_{0}^{\tau}\left|\widetilde{w}_{1}(t)-\widetilde{w}_{2}(t)\right|^{2} d t, \quad \tau \in(0, T] .
\end{gathered}
$$

Multiplying (38) by $e^{-2 \alpha \tau}$, where $\alpha>1$ is some constant, we obtain

$$
\begin{gathered}
e^{-2 \alpha \tau}\left|w_{1}(\tau)-w_{2}(\tau)\right|^{2} \leq \\
\leq 2 e^{-2 \alpha \tau} \int_{0}^{\tau} e^{2 \alpha t} e^{-2 \alpha t}\left|w_{1}(t)-w_{2}(t)\right|^{2} d t+ \\
+2^{-1}(L T)^{2} e^{-2 \alpha \tau} \int_{0}^{\tau} e^{2 \alpha t} e^{-2 \alpha t}\left|\widetilde{w}_{1}(t)-\widetilde{w}_{2}(t)\right|^{2} d t \leq \\
\leq 2 e^{-2 \alpha \tau} \max _{t \in[0, T]}\left[e^{-2 \alpha t}\left|w_{1}(t)-w_{2}(t)\right|^{2}\right] \int_{0}^{\tau} e^{2 \alpha t} d t+
\end{gathered}
$$

$$
\begin{aligned}
& +2^{-1}(L T)^{2} e^{-2 \alpha \tau} \max _{t \in[0, T]}\left[e^{-2 \alpha t}\left|\widetilde{w}_{1}(t)-\widetilde{w}_{2}(t)\right|^{2}\right] \int_{0}^{\tau} e^{2 \alpha t} d t= \\
& =(1 / \alpha)\left(1-e^{-2 \alpha \tau}\right)\left(\rho\left(w_{1}, w_{2}\right)\right)^{2}+ \\
& +\left((L T)^{2} / 4 \alpha\right)\left(1-e^{-2 \alpha \tau}\right)\left(\rho\left(\widetilde{w}_{1}, \widetilde{w}_{2}\right)\right)^{2} \leq \\
& \leq(1 / \alpha)\left(\rho\left(w_{1}, w_{2}\right)\right)^{2}+\left((L T)^{2} /(4 \alpha)\right)\left(\rho\left(\widetilde{w}_{1}, \widetilde{w}_{2}\right)\right)^{2}, \quad(39) \\
& \tau \in[0, T] .
\end{aligned}
$$

From (38) it follows

$$
\rho\left(w_{1}, w_{2}\right) \leq(L T /(2 \sqrt{\alpha-1})) \rho\left(\widetilde{w}_{1}, \widetilde{w}_{2}\right) .
$$

Thus, choosing $\alpha>1$ such that $L T /(2 \sqrt{\alpha-1})<1$, we obtain, that operator $A$ is a contraction operator. Hence, we may apply the Banach fixed point theorem (the contraction mapping principle) [6, theorem 5.7], and deduce that there exists a unique function $u \in M$ such that $A u=u$, i.e., there exists a unique solution $u$ of the problem $\mathbf{P}\left(\Phi_{H}, \mathcal{B}, f, u_{0}\right)$ (see (25)).

Let us show that $u$ is a solution of the problem $\mathbf{P}\left(\Phi, \mathcal{B}, f, u_{0}\right)$. Indeed, taking into account that $D\left(\partial \Phi_{H}\right) \subset$ $\operatorname{dom}\left(\Phi_{H}\right)=\operatorname{dom}(\Phi)$, we obtain that $u(t) \in V$ for a.e. $t \in(0, T)$. According to the definition of the subdifferential of the functional and the fact that $g(t) \in \partial \Phi_{H}(u(t))$ for a.e. $t \in(0, T)$ we have

$$
\Phi(0) \geq \Phi(u(t))+(g(t), 0-u(t)) \text { for a.e. } t \in(0, T) .
$$

This and condition $\left(\mathcal{A}_{3}\right)$ yields that for a.e. $t \in(0, T)$ we have

$$
(g(t), u(t)) \geq \Phi(u(t)) \geq K\|u(t)\|^{p} .
$$

Since the left side of this chain of inequalities belongs to $L^{1}(0, T), u$ belongs to $L^{p}(0, T ; V)$. Thus, $u$ is a solution of the problem $\mathbf{P}\left(\Phi, \mathcal{B}, f, u_{0}\right)$.

Let us prove now the accuracy of estimates (15) and (16).

Multiplying (25) by $u(t)$ and integrating by $t$ from 0 to $\tau \in$ $(0, T]$ we obtain

$$
\begin{aligned}
\int_{0}^{\tau}\left(u^{\prime}(t), u(t)\right) d t+ & \int_{0}^{\tau}(g(t), u(t)) d t+\int_{0}^{\tau}(\mathcal{B}(u)(t), u(t)) d t= \\
& =\int_{0}^{\tau}(f(t), u(t)) d t .
\end{aligned}
$$

From this, using equality (6), we get

$$
\begin{gathered}
\int_{0}^{\tau} \frac{d}{d t}|u(t)|^{2} d t+2 \int_{0}^{\tau}(g(t), u(t)) d t+2 \int_{0}^{\tau}(\mathcal{B}(u)(t), u(t)) d t= \\
=2 \int_{0}^{\tau}(f(t), u(t)) d t
\end{gathered}
$$

Integrating by parts the first term of the left-hand side of the equality (41) we obtain

$$
\begin{gathered}
|u(\tau)|^{2}+2 \int_{0}^{\tau}(g(t), u(t)) d t+2 \int_{0}^{\tau}(\mathcal{B}(u)(t), u(t)) d t= \\
=\left|u_{0}\right|^{2}+2 \int_{0}^{\tau}(f(t), u(t)) d t .
\end{gathered}
$$


By using (40), we estimate now the second term of the lefthand side of the equality (42)

$$
\begin{gathered}
2 \int_{0}^{\tau}(g(t), u(t)) d t \geq \\
\geq \int_{0}^{\tau} \Phi(u(t)) d t+K \int_{0}^{\tau}\|u(t)\|^{p} d t .
\end{gathered}
$$

Let us estimate the third term of the left-hand side of (42) as follows

$$
\begin{gathered}
2\left|\int_{0}^{\tau}(\mathcal{B}(u)(t), u(t)) d t\right| \leq 2 L \int_{0}^{\tau}\left(\int_{0}^{t}|u(s)| d s\right)|u(t)| d t \leq \\
\leq 2 L \sqrt{T}\left(\int_{0}^{\tau}|u(t)|^{2} d t\right)^{1 / 2}\left(\int_{0}^{\tau}\left(\int_{0}^{t}|u(s)|^{2} d s\right) d t\right)^{1 / 2} \leq \\
\leq 2 L T \int_{0}^{\tau}|u(t)|^{2} d t .
\end{gathered}
$$

By using the Cauchy-Schwartz inequality (34), let us estimate the second term of the right-hand side of the equality (42). As a result we obtain

$$
\begin{gathered}
2 \int_{0}^{\tau}(f(t), u(t)) d t \leq 2 \int_{0}^{\tau}|f(t)||u(t)| d t \leq \\
\leq \int_{0}^{\tau}|u(t)|^{2} d t+\int_{0}^{\tau}|f(t)|^{2} d t .
\end{gathered}
$$

From (42) by using (43), (44), and (45), we get

$$
\begin{gathered}
|u(\tau)|^{2}+K \int_{0}^{\tau}\|u(t)\|^{p} d t+\int_{0}^{\tau} \Phi(u(t)) d t \leq \\
\leq\left|u_{0}\right|^{2}+2 L T \int_{0}^{\tau}|u(t)|^{2} d t+\int_{0}^{\tau}|u(t)|^{2} d t+\int_{0}^{\tau}|f(t)|^{2} d t \leq \\
\leq\left|u_{0}\right|^{2}+\int_{0}^{T}|f(t)|^{2} d t+(2 L T+1) \int_{0}^{\tau}|u(t)|^{2} d t, \quad \text { (46) }
\end{gathered}
$$

$\tau \in(0, T]$.

Using (46) and the Gronwall-Belman Lemma, we obtain

$$
|u(\tau)|^{2} \leq C_{3}\left(\left|u_{0}\right|^{2}+\int_{0}^{T}|f(t)|^{2} d t\right),
$$

where $C_{3}>0$ is a constant depended on $L, T$ only.

Based on (47), we get

$$
\int_{0}^{\tau}|u(t)|^{2} d t \leq C_{3} \int_{0}^{\tau}\left(\left|u_{0}\right|^{2}+\int_{0}^{T}|f(s)|^{2} d s\right) d t \leq
$$

$$
\leq C_{4}\left(\left|u_{0}\right|^{2}+\int_{0}^{T}|f(t)|^{2} d t\right),
$$

where $C_{4}:=T C_{3}$.

From (46) and (48) by virtue of arbitrariness $\tau \in[0, T]$ we obtain

$$
\begin{gathered}
\max _{t \in[0, T]}|u(t)|^{2}+K \int_{0}^{T}\|u(t)\|^{p} d t+\int_{0}^{T} \Phi(u(t)) d t \leq \\
\leq C_{5}\left(\left|u_{0}\right|^{2}+\int_{0}^{T}|f(t)|^{2} d t\right)
\end{gathered}
$$

where $C_{5}$ is a positive constant depended on $L$ and $T$ only.

From this it follows (15).

Let us find an estimate of function $u^{\prime}$. For almost every $t \in(0, T)$ we multiply (25) by $u^{\prime}(t)$ and integrate by $t$ from 0 to $\tau \in(0, T]$. Then we obtain

$$
\begin{gathered}
\int_{0}^{\tau}\left|u^{\prime}(t)\right|^{2} d t+\int_{0}^{\tau}\left(g(t), u^{\prime}(t)\right) d t+\int_{0}^{\tau}\left(\mathcal{B}(u)(t), u^{\prime}(t)\right) d t= \\
=\int_{0}^{\tau}\left(f(t), u^{\prime}(t)\right) d t .
\end{gathered}
$$

Since $g \in L^{2}(0, T ; H)$ and $g(t) \in \partial \Phi_{H}(u(t))$ for a.e. $t \in(0, T)$, Proposition 2 implies that the function $\Phi_{H}(u(\cdot))$ is absolutely continuous on $[0, T]$ and

$$
\frac{d}{d t} \Phi_{H}(u(t))=\left(g(t), u^{\prime}(t)\right) \text { for a.e. } t \in(0, T) .
$$

By (51) we can rewrite the second term of the left-hand side of equality (50) as follows

$$
\begin{gathered}
\int_{0}^{\tau}\left(g(t), u^{\prime}(t)\right) d t=\int_{0}^{\tau} \frac{d}{d t} \Phi_{H}(u(t)) d t= \\
=\Phi_{H}(u(\tau))-\Phi_{H}(u(0)) .
\end{gathered}
$$

Let us evaluate the third term of the left-hand side of equality (50). By using (8) and the Cauchy inequality (34) we obtain

$$
\begin{gathered}
\left|\int_{0}^{\tau}\left(\mathcal{B}(u)(t), u^{\prime}(t)\right) d t\right| \leq \int_{0}^{\tau}|\mathcal{B}(u)(t)|\left|u^{\prime}(t)\right| d t \leq \\
\leq L \int_{0}^{\tau}\left(\int_{0}^{t}|u(s)| d s\right)\left|u^{\prime}(t)\right| d t \leq \\
\leq L^{2} \int_{0}^{\tau}\left(\int_{0}^{t}|u(s)| d s\right)^{2} d t+4^{-1} \int_{0}^{\tau}\left|u^{\prime}(t)\right|^{2} d t \leq \\
\leq L^{2} T \int_{0}^{\tau}\left(\int_{0}^{t}|u(s)|^{2} d s\right) d t+4^{-1} \int_{0}^{\tau}\left|u^{\prime}(t)\right|^{2} d t \leq \\
\leq(L T)^{2} \int_{0}^{\tau}|u(t)|^{2} d t+4^{-1} \int_{0}^{\tau}\left|u^{\prime}(t)\right|^{2} d t .
\end{gathered}
$$


Let us estimate the right-hand side of the equality (50) by using the Cauchy inequality (34) as follows

$$
\begin{gathered}
\int_{0}^{\tau}\left(f(t), u^{\prime}(t)\right) d t \leq \\
\leq \int_{0}^{\tau}|f(t)|^{2} d t+4^{-1} \int_{0}^{\tau}\left|u^{\prime}(t)\right|^{2} d t .
\end{gathered}
$$

By (52) - (54), from (50) we get

$$
\begin{gathered}
\frac{1}{2} \int_{0}^{\tau}\left|u^{\prime}(t)\right|^{2} d t+\Phi_{H}(u(\tau)) \leq \\
\leq \Phi_{H}\left(u_{0}\right)+(L T)^{2} \int_{0}^{\tau}|u(t)|^{2} d t+\int_{0}^{\tau}|f(t)|^{2} d t .
\end{gathered}
$$

Taking into account estimate (48), from (55) we have

$$
\begin{gathered}
\int_{0}^{\tau}\left|u^{\prime}(t)\right|^{2} d t+\Phi_{H}(u(\tau)) \leq \\
\leq \Phi_{H}\left(u_{0}\right)+C_{6}\left(\left|u_{0}\right|^{2}+\int_{0}^{T}|f(t)|^{2} d t\right),
\end{gathered}
$$

where $C_{6}$ is a positive constant depended on $K, L$, and $T$ only.

According to the definitions of the functional $\Phi_{H}$, condition $\left(\mathcal{A}_{3}\right)$ (recall that $u(t) \in V$ for a.e. $t \in[0, T]$ ) and an arbitrariness of $\tau \in[0, T]$ from (56) we have

$$
\begin{gathered}
\underset{t \in[0, T]}{\operatorname{ess} \sup _{t}}\|u(t)\|^{p}+\max _{t \in[0, T]} \Phi(u(t))+\int_{0}^{T}\left|u^{\prime}(t)\right|^{2} d t \leq \\
\quad \leq C_{7}\left[\Phi\left(u_{0}\right)+C_{6}\left(\left|u_{0}\right|^{2}+\int_{0}^{T}|f(t)|^{2} d t\right)\right],
\end{gathered}
$$

where $C_{7}$ is a positive constant depended on $K, T$, and $L$ only.

From this it follows (16).

\section{Conclusions}

This paper concerns the initial-value problems for parabolic variational inequalities (sub-differential inclusions) with Volterra type operators. We prove that there exists no more than one weak solution of the considered problem. The results are achieved using the Banach's fixed point theorem, that is, the principle of compression mappings. Furthermore, the estimates of the solution are obtained.

\section{Acknowledgements}

The authors sincerely thank the referees for their valuable comments.

\section{REFERENCES}

[1] J.-L. Lions. Quelques méthodes de résolution des problémes aux limites non linéaires, Paris (France): Dunod GauthierVillars, 1969.

[2] H. Brezis, D. Kinderlehrer. The smoothness of solutions to nonlinear variational inequalities, Indiana Univ. Math. J., Vol. 17, 831-844, 1974.

[3] A. Kovalevsky, F. Nicolosi. Boundness of solutions of degenerate nonlinear elliptic variational inequalities, Nonlinear Anal., Theory Methods Appl., Vol. 35, No. 8, 987-999, 1999.

[4] J.-P. Aubin. Un theoreme de compacite, Comptes rendus hebdomadaires des seances de l'academie des sciences, Vol. 256, No. 24, 5042-5044, 2007.

[5] M. Bokalo. Well-posedness of problems without initial conditions for nonlinear parabolic variational inequalities, Nonlinear boundary problem, Vol. 8, 58-63, 1998.

[6] H. Brezis. Functional Analysis, Sobolev Spaces and Partial Differential Equations, Springer New York Dordrecht Heidelberg London, (2011).

[7] H. Brézis. Opérateurs maximaux monotones et semi-groupes de contractions dans les espaces de Hilbert, Amsterdam, London: North-Holland Publishing Comp., 1973.

[8] O. Buhrii. Some parabolic variational inequalities without initial conditions, Visnyk of the Lviv Univ. Series Mech. Math., Vol. 49, 113-121, 1998.

[9] M. Barboteu, J. R. Fernandez, T.-V. Hoarau-Mantel. A class of evolutionary variational inequalities with applications in viscoelasticity, Mathematical Models and Methods in Applied Sciences, Vol. 15, No. 10, 1595-1617, 2005.

[10] H. Gayevskyy, K. Greger, K. Zaharias. Nonlinear operator equations and operator differential equations, Moscow, Mir, 1978.

[11] R. Rockafellar. On the maximal monotonicity of subdifferential mappings, Pacific J. Math., Vol. 33, No. 1, 209-216, 1970.

[12] R. Showalter. Monotone operators in Banach space and nonlinear partial differential equations, Mathematical Surveys and Monographs,Providence: Amer. Math. Soc., Vol. 49, 1997.

[13] K. Yoshida. Functional Analysis, [Russian translation], Mir, Moscow, 1967.

[14] D. Pascali, S. Sburlan. Nonlinear mappings of monotone type, Sijthoff and Noordhoff, Bucharest, 1978.

[15] R. Mashiyev, O. Buhrii. Existence of solutions of the parabolic variational inequality with variable exponent of nonlinearity, J. Math. Anal. Appl., Vol. 377, 450-463, 2011.

[16] O. Buhrii. Parabolic variational inequalities with degeneration, Mat. stud., Vol. 11, No. 2, 189-198, 1999.

[17] M. Bokalo, O. Sus. Initial-boundary value problems for nonlinear degenerate parabolic equations with integral operators type Volterra, Visnyk of the Lviv Univ. Series Mech. Math., Vol. 82, 56-74, 2016. 
[18] M. Bokalo, O. Sus. Initial-boundary value problems for nonlinear parabolic equations with the variable exponents of nonlinearity and integral operators type Volterra, Research in mathematics and mechanics, Vol. 21(2), No. 28, 19-36, 2016.

[19] S. Carl, S. Heikkila, J.W. Jerome. Trapping regions for discontinuously coupled systems of evolution variational inequalities and application, J. Math. Anal. Appl., Vol. 282, 421—435, 2003.

[20] J. Jarusek, M. Krbec, M. Rao, J. Sokolowski. Conical differentiability for evolution variational inequalities, J. Differential Equations, Vol. 193, 131-146, 2003.

[21] M. Bokalo, A. Tsebenko. Optimal control in problems without initial conditions for evolution variational inequalities, Mat. stud., Vol. 46, No. 1, 51-66, 2016.

[22] M. Bokalo, A. Tsebenko. Optimal control in problems without initial conditions for weakly nonlinear evolution variational inequalities, Visnyk of the Lviv Univ. Series Mech. Math., Vol. 82, 76-94, 2016.

[23] S. P. Lavrenyuk. Parabolic variational inequalities without initial conditions, J. Differential equations, Vol. 32, No. 10, 1-5, 1996.

[24] S. P. Lavrenyuk. Systems of parabolic variational inequalities without initial conditions, Ukrain. Math. Journal, Vol. 49, No. 4, 540-547, 1997.
[25] L. Gasinski, S. Migorski, A. Ochal. Existence results for evolutionary inclusions and variational-hemivariational inequalities, Applicable Analysis An International Journal, Vol. 94, No. 8, 1670-1694, 2014.

[26] S. Migorski, A. Ochal, M. Sofonea. Evolutionary Inclusions and Hemivariational Inequalities, Advances in mechanics and mathematics: Kluwer Academic Springer, Vol. 33, 39-64, 2015.

[27] M. Sofonea, A. Matei. Volterra-type Variational Inequalities, In: Variational Inequalities with Applications. Advances in Mechanics and Mathematics, Vol. 18, Springer, New York, NY, 2009.

[28] A. Rodrlguez-Aros, M. Sofonea, J.M. Viafio. A class of evolutionary variational inequalities with Volterra type integral term, Mathematical Models and Methods in Applied Sciences, Vol. 14, 555-577, 2004.

[29] A. Rodriguez-Aros, M. Sofonea, J.M. Viafio. Numerical analysis of a class of evolutionary variational inequalities with integral term, Ann. Univ. Craiova, Math. Comp. Sci. Series, Vol. 30, 30-37, 2003.

[30] J. Steinbach. Evolutionary variational inequalities with a Volterra term, Numerical Functional Analysis and Optimization Taylor and Francis, Vol. 12, No. (3-4), 395-411, 1991. 